\title{
Spin-Wave Band Structure in 2D Magnonic Crystals with Elliptically Shaped Scattering Centres
}

\author{
Sławomir Mamica, Maciej Krawczyk, and Jarosław Wojciech Kłos \\ Nanomaterials Physics Division, Faculty of Physics, Adam Mickiewicz University, 61-614 Poznań, Poland \\ Correspondence should be addressed to Sławomir Mamica, mamica@amu.edu.pl
}

Received 2 February 2012; Accepted 2 March 2012

Academic Editor: Roberto Zivieri

Copyright (C) 2012 Sławomir Mamica et al. This is an open access article distributed under the Creative Commons Attribution License, which permits unrestricted use, distribution, and reproduction in any medium, provided the original work is properly cited.

Spin waves in 2D periodic magnetic nanocomposites are studied by means of the plane wave method. The effect of the ellipticity and in-plane rotation of the scattering centers on the band structure is investigated, to indicate new possibilities of fine tuning of spin-wave filter passbands.

\section{Introduction}

Magnetic composites with a structure modulated periodically on the nanoscale are the subject of a very intensive research activity, and the interest in their properties is increasing [1-7]. By analogy to photonic crystals (PCs), they are referred to as magnonic crystals (MCs) [8], since the role of information carriers in these materials is played by magnons, or spin-wave quanta. Magnonic crystals have properties that cannot be reduced to those of their constituent materials, as manifested, among others, by the band structure of their spin-wave spectrum. The periodic structure given to a magnetic composite strictly determines the possible occurrence of magnonic gaps, or energy ranges forbidden to propagating spin waves [9-11]. The anticipated full control over the spin waves propagating in MCs, similar to that of the electromagnetic waves in PCs, combined with a long-term stability of the programmable magnetic state makes MCs excellent for both research and application purposes $[12,13]$. Moreover, the wavelength of spin waves is much shorter than that of electromagnetic waves of the same frequency. This provides additional possibilities in the miniaturization of MC-based devices [14-16]. For a broad survey of the current state of both the experimental and theoretical research in MCs and their potential applications, please refer to review papers $[17,18]$.

The most frequently mentioned of the numerous potential applications of MCs include microwave resonators, magnonic waveguides, spin-wave emitters, and filters [19]. These potentia applications, along with the possibility of modeling the energy spectrum of spin-wave excitations propagating in MCs, are the very reason of the intensification of the research on new magnonic materials with so far unknown properties and functionalities $[14,16]$. In particular, two-dimensional (2D) MCs with band gaps in the spin-wave spectrum have potential applications in diverse magnonic devices, such as spin-wave filters or switches, or current-controlled delay lines [20]. For example, the latest results of micromagnetic simulations [21] indicate the occurrence of wide magnonic gaps, implying a possible application in spin-wave filters, in 2D Fe/YIG MCs, twocomponent magnetic composites with iron scattering centers embedded in a matrix of yttrium iron garnet. A particular role in the modeling of magnonic gaps is played by the deformation of the scattering centers in the plane of spinwave propagation [22].

In this study we examine the possibilities of modeling the spin-wave spectrum of 2D MCs that could be used for fine tuning of spin-wave filter passbands [23]. We present the results of calculations of the magnonic band structure of a $\mathrm{Co} / \mathrm{Fe}$ composite with scattering centers in the shape of elliptic cylinders. We find that for different filling fraction values there are specific in-plane rotation angles for which modifying the rod ellipticity can alter the position of the allowed band without changing its width or 
cause a substantial shrinking of two adjacent bands without changing the width of the gap between them.

\section{The Model}

Figure 1(a) depicts schematically a section of the system under consideration in the plane perpendicular to the rod axis (the $x-y$ plane), which is the plane of spin-wave propagation. The system includes cobalt rods (scattering centers), which are assumed to be parallel to each other and have an infinite length. The rods are arranged in sites of a $2 \mathrm{D}$ square lattice and embedded in an iron matrix. The system is infinite in the plane of periodicity. The filling fraction $f f$, describing the proportion of the rod material in the whole volume of the system, in the case of elliptic cylinders is given as $f f=\pi R_{a} R_{b} / a^{2}$, where $R_{a}$ and $R_{b}$ are the semiaxes of the rod cross section, and $a$ is the lattice constant. Let us define the ellipticity $R R$ of the rods as the semiaxis ratio: $R R=R_{a} / R_{b}$. The angle $\alpha$ between the major semiaxis $R_{a}$ and the $x$-axis of the crystallographic system is the angle of rotation of the rods (in the plane of periodicity). Applied to the system, an external magnetic field perpendicular to the plane of periodicity is assumed to be strong enough to enforce a uniform magnetization throughout the system.

We shall consider in-plane propagation of spin waves, that is, their propagation in the plane of periodicity, in 2D MCs as described above. Thus, the wave vectors to be considered are limited to the Brillouin zone of the $2 \mathrm{D}$ lattice. Figure 1(b) shows the high-symmetry line in such a 2D Brillouin zone. In the case of square lattice the line starts at the zone center (point $\Gamma$ ) to pass through $X$ and $M$ and return to $\Gamma$ (segments $C, D$, and $E$ in Figure $1(\mathrm{~b})$ ). The introduction of rods with elliptical cross section breaks the symmetry of the square lattice. If the semiaxes of the ellipse follow the axes of the 2D crystallographic system, the structure has the symmetry of a rectangular lattice. Points $X$ and $X^{\prime}$ are not equivalent anymore, and the high-symmetry line in this case leads from $M$ to $X^{\prime}, \Gamma, X$, and back to $M$ (segments $A, B, C$, and $D$ ). For an ellipse rotated by $45^{\circ}$ points, $X$ and $X^{\prime}$ are equivalent symmetry points, whereas $M$ and $M^{\prime}$ are not. Thus, the high-symmetry line leads from $\Gamma$ to $X, M, \Gamma, M^{\prime}$, and $X$ (segments $C, D, E, F, G$ ). In the general case, that is, for any angle of rotation of the ellipse, the full line shown in Figure 1(b) must be considered.

Our theoretical approach is based on a set of equations including the linearized Landau-Lifshitz equation and Maxwell's magnetostatic equations [24]. When the applied magnetic field is parallel to the rods, the internal static magnetic field is uniform, which allows us to only take into account the exchange and dynamic dipolar interactions. The ferromagnetic materials of the rods and the matrix are characterized by two quantities: the spontaneous magnetization $M_{s}$ and the exchange stiffness constant $A$. For the materials considered in this study the specific values of these two magnetic parameters are for iron $M_{s}=1.752 e 6 \mathrm{~A} / \mathrm{m}$ and $A=2.1 e-11 \mathrm{~J} / \mathrm{m}$, and for cobalt $M_{s}=1.390 e 6 \mathrm{~A} / \mathrm{m}$ and $A=2.8 e-11 \mathrm{~J} / \mathrm{m}$ [25]. Crucial for the magnonic nature of the structure under consideration is the assumption that these two material parameters are periodic functions of position, with the same periodicity as the $2 \mathrm{D}$ lattice on which the magnonic crystal is built. With this assumption our equations can be solved by the plane wave method. The main point of this method is the Fourier expansion of the material parameters. Bloch theorem is applied to the dynamic functions, such as the demagnetizing field potential and the dynamic component of magnetization. Thus, the equations are transformed to the reciprocal space, where their solution is equivalent to the diagonalization of a $2 \mathrm{~N} \times$ $2 N$ matrix, $N$ being the number of plane waves used in the Fourier expansion (for more details, see [24] and references therein).

\section{The Role of Magnetostatic Interactions}

Figure 2 shows examples of the so-called magnonic spectra, represented by the spin-wave spectra of $2 \mathrm{D} \mathrm{Co} / \mathrm{Fe}$ magnetic composites, calculated along the high-symmetry line in the 2D Brillouin zone. The spectra shown in Figures 2(a) and 2(b) have been obtained for lattice constants $a=50 \mathrm{~nm}$ and $a=100 \mathrm{~nm}$, respectively. A circular cross section of the $\operatorname{rods}(R R=1)$ and a filling fraction $f f=0.5$ are assumed in both cases. For small lattice constants the exchange interactions play a dominant role; however, as the lattice constant grows, their importance diminishes to the advantage of the magnetostatic interactions [26, 27]. As a result, the spin-wave frequency range lowers; for the lattice constant of $50 \mathrm{~nm}$ the frequency of the ten lowest modes is below $80 \mathrm{GHz}$ (Figure 2(a)), but only ranges from $10 \mathrm{GHz}$ to $35 \mathrm{GHz}$ for $a=100 \mathrm{~nm}$ (Figure 2(b)). The flattening of successive bands results in the opening of a magnonic gap: for $a=50 \mathrm{~nm}$ all the bands overlap, while for $a=100 \mathrm{~nm}$ a gap occurs between the lowest band and the rest of the spectrum.

\section{The Ellipticity and Rotation of the Rods}

Figure 3 presents the effect of the cross-sectional ellipticity of the scattering centers and their rotation in the plane of spin-wave propagation on the magnonic spectrum in a $\mathrm{Co} / \mathrm{Fe}$ composite with a lattice constant of $300 \mathrm{~nm}$ and a filling fraction of 0.3 . For unrotated rods (Figure 3(a)) the maximum ellipticity (corresponding to touching rods) is $R R=2.6$, which means the major and minor semiaxes $R_{a}$ and $R_{b}$ can range from $92.7 \mathrm{~nm}$ up to $149.8 \mathrm{~nm}$ and down to $57.4 \mathrm{~nm}$, respectively. In the whole range of rod ellipticity the bottom of the lowest band is nearly constant, remaining between $10.84 \mathrm{GHz}$ and $10.89 \mathrm{GHz}$. Also the top of the second band varies very slightly, only ranging from 12.04 GHz to $12.15 \mathrm{GHz}$. However, the width of both bands grows rapidly, as the top of the first band and the bottom of the second one converge. Consequently, the gap between the bands shrinks to vanish completely for $R R=2.1\left(R_{a}=\right.$ $\left.134.3 \mathrm{~nm}, R_{b}=64.0 \mathrm{~nm}\right)$. The two bands merge to form a single wide band, separated from the rest of the spectrum by a third gap, the width of which varies from $1.26 \mathrm{GHz}$ for right circular cylinders $(R R=1.0)$ to $0.79 \mathrm{GHz}$ for $R R=2.1$ (the closing of the second gap) to $0.37 \mathrm{GHz}$ for $R R=2.6$ (the 


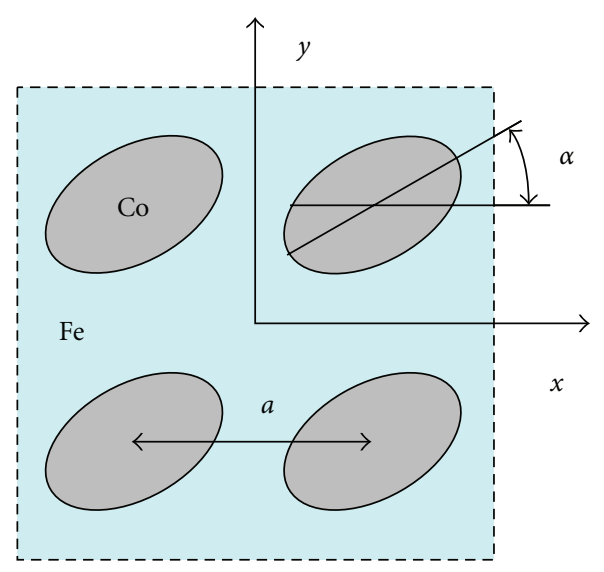

(a)

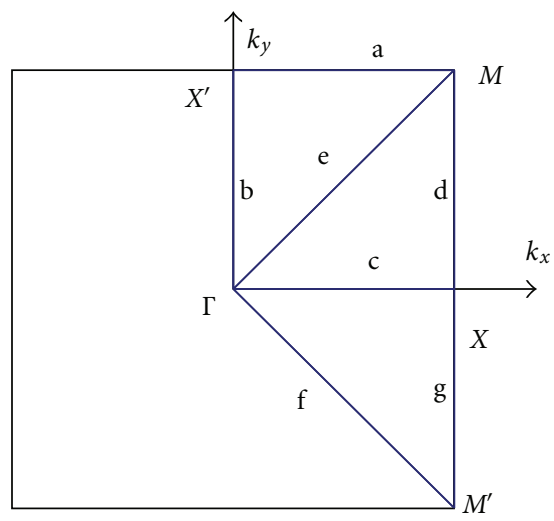

(b)

FIgURE 1: (a) Schematic view of the 2D MC under consideration, section in the plane of periodicity. (b) High-symmetry line over the 2D Brillouin zone for ellipses arranged in a square lattice.

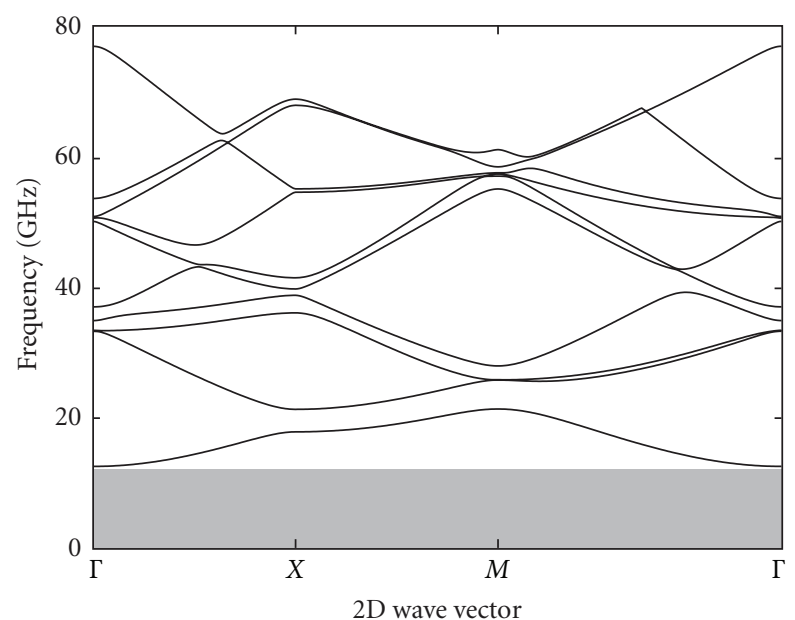

(a)

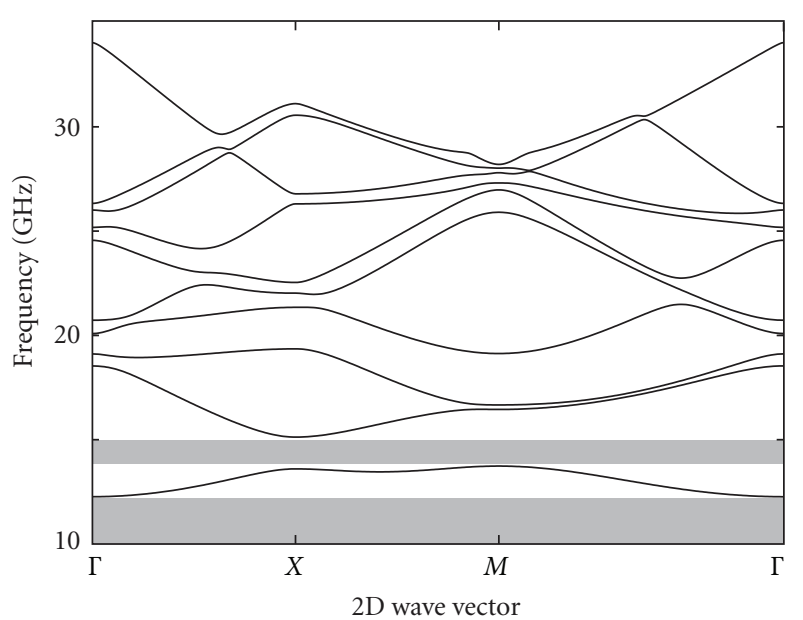

(b)

Figure 2: Ten lowest bands in the spin-wave spectrum of a 2D Co/Fe composite with lattice constant (a) $50 \mathrm{~nm}$ and (b) $100 \mathrm{~nm}$, calculated along the high-symmetry line in the 2D Brillouin zone (cf. Figure 1(b)). Circular cross section of the rods $(R R=1)$ and filling fraction $f f=0.5$ are assumed in both cases. Shaded areas represent magnonic gaps. Note the plots differ in frequency range: $0 \mathrm{GHz}$ to $80 \mathrm{GHz}$ in (a), and $10 \mathrm{GHz}$ to $35 \mathrm{GHz}$ in (b).

maximum ellipticity). Figure 3(b) shows the band and gap widths plotted versus the rod ellipticity.

Also for rods rotated by $45^{\circ}$ (Figure $3(\mathrm{c})$ ) the second gap is seen to shrink, though not as rapidly as in the case of unrotated rods. The gap closes for $R R=3.45$, which corresponds to $R_{a}=212.0 \mathrm{~nm}$ and $R_{b}=40.5 \mathrm{~nm}$. A significant difference with respect to the composite with unrotated rods is seen in the behavior of the second band, which moves down the frequency scale with nearly constant width as $R R$ grows from 1.0 to 2.8 (cf. Figure $3(\mathrm{~d})$ ).

In a $\mathrm{Co} / \mathrm{Fe}$ composite with a filling fraction of 0.5 and rods unrotated in the plane of periodicity (the major semiaxis following the $x$ direction), the rod ellipticity can range from 1.0 (circular cross section) to 1.57, which for the assumed lattice constant $a=300 \mathrm{~nm}$ corresponds to the major semiaxis ranging from $119.7 \mathrm{~nm}$ up to $150.0 \mathrm{~nm}$, and the minor semiaxis from $119.7 \mathrm{~nm}$ down to $95.5 \mathrm{~nm}$. As the ellipticity of the cylinders grows, the gaps are seen to shrink and the bands to widen (Figure 4(a)). Although the bottom of the lowest band at first moves towards higher frequencies, the change is to slight to compensate the concurrent rising of the top. In the second band the top is seen to descend slightly, while the bottom moves much faster in the same direction. As a consequence, the second gap, between the first and second bands, shrinks rapidly with growing ellipticity to vanish completely for $R R=1.2\left(R_{a}=131.1 \mathrm{~nm}, R_{b}=\right.$ $109.3 \mathrm{~nm})$. The first and second bands merge to form one relatively wide band (of width ranging from $1.17 \mathrm{GHz}$ for $R R=1.2$ to $1.32 \mathrm{GHz}$ for $R R=1.57$ ), separated from the rest of the spectrum by a third gap, which has a maximum width of $0.65 \mathrm{GHz}$ for $R R=1.2$, and a minimum width of $0.25 \mathrm{GHz}$ for $R R=1.57$ (cf. Figure $4(\mathrm{~b})$ ). 


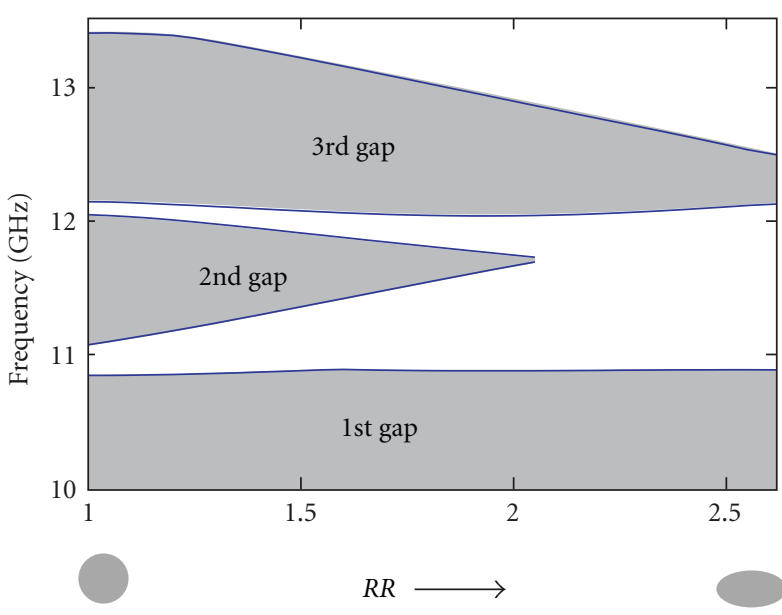

(a)

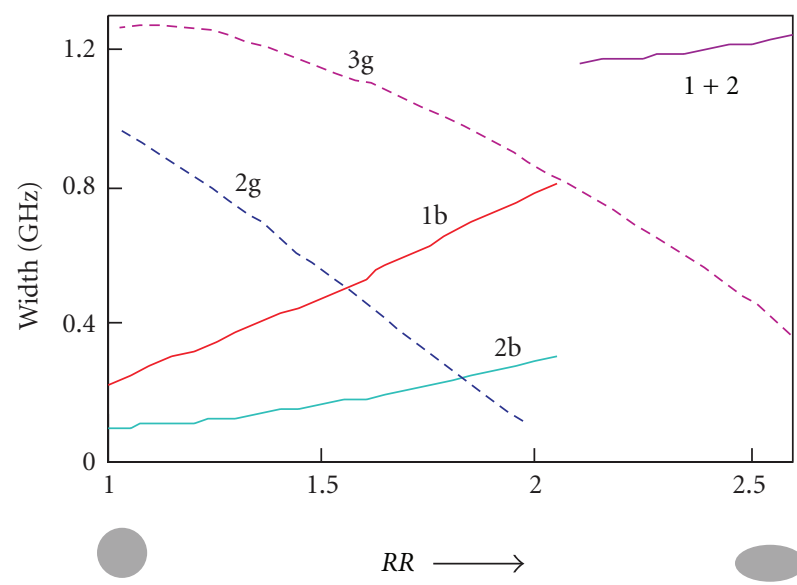

(b)

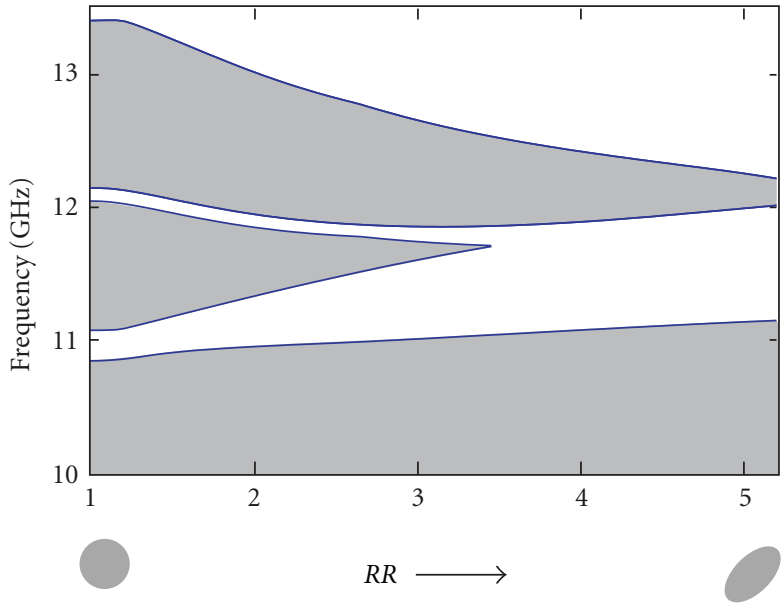

(c)

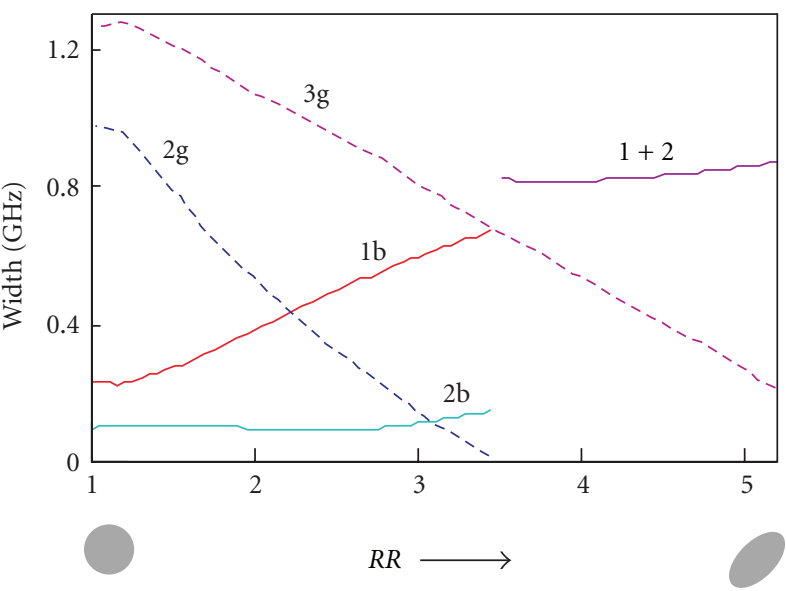

(d)

Figure 3: (a), (c) Three lowest magnonic gaps (shaded) versus rod ellipticity $R R$ for 2D Co/Fe MCs with lattice constant 300 nm, filling fraction 0.3, and two angles of in-plane rotation of the rods: (a) $\alpha=0^{\circ}$ and (c) $\alpha=45^{\circ}$. (b), (d) The width of the lowest gaps and bands versus $R R$ in (a) and (c), respectively. The width of the second $(2 \mathrm{~g})$ and third $(3 \mathrm{~g})$ gaps is plotted with dashed line. Solid line represents the width of the lowest bands: the first (1b), the second (2b), and, in the range of $R R$ values in which the gap between them does not occur, the band resulting from their merging $(1+2)$.

A completely different behavior of the spin-wave spectrum is seen for the same filling fraction $(f f=0.5)$, but with rods rotated by $45^{\circ}$ in the plane of periodicity (Figure 4(c)). Two ranges of $R R$ can be distinguished in this case. In the first range, from 1.0 to 2.0 (the major semiaxis growing from $119.7 \mathrm{~nm}$ to $169.3 \mathrm{~nm}$, and the minor semiaxis shrinking from $119.7 \mathrm{~nm}$ to $84.6 \mathrm{~nm}$ ), the width of both lowest bands decreases substantially, the first one shrinking from $0.52 \mathrm{GHz}$ to $0.28 \mathrm{GHz}$, and the second from $0.34 \mathrm{GHz}$ to $0.22 \mathrm{GHz}$ (Figure $4(\mathrm{~d})$ ). At the same time, the midlevels of both bands move, from $11.0 \mathrm{GHz}$ to $11.1 \mathrm{GHz}$ in the case of the first band, and from $11.7 \mathrm{GHz}$ to $11.6 \mathrm{GHz}$ for the second. Interestingly, in this range of $R R$ the second gap remains nearly unchanged, its width only ranging from $0.29 \mathrm{GHz}$ to $0.30 \mathrm{GHz}$. In the other part of the $R R$ dependence the spectrum behaves as in the cases considered previously: the bands widen and the gaps shrink rapidly. However, in this case even for the maximum ellipticity of the rods $(R R=3.14)$ the second gap will not close completely, though its width falls as low as $0.02 \mathrm{GHz}$.

\section{Conclusions}

In the $2 \mathrm{D}$ magnetic composites considered in this paper, with $\mathrm{Co}$ rods embedded in an $\mathrm{Fe}$ matrix, the increase in importance of the magnetostatic interactions results in the formation of band gaps in the spin-wave spectrum. These magnonic gaps are destroyed as the exchange interactions begin to play a dominant role. On the other hand, the increase in importance of the exchange interactions not only results in a widening of the bands, but also, consequently, causes the possible gaps to move towards higher frequencies. In contrast, when the magnetostatic interactions gain in importance at the cost of the exchange interactions, the 


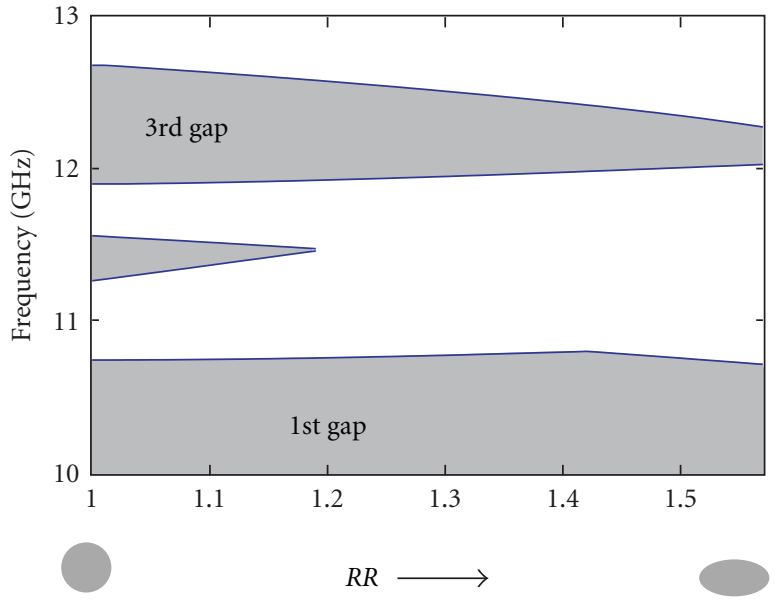

(a)

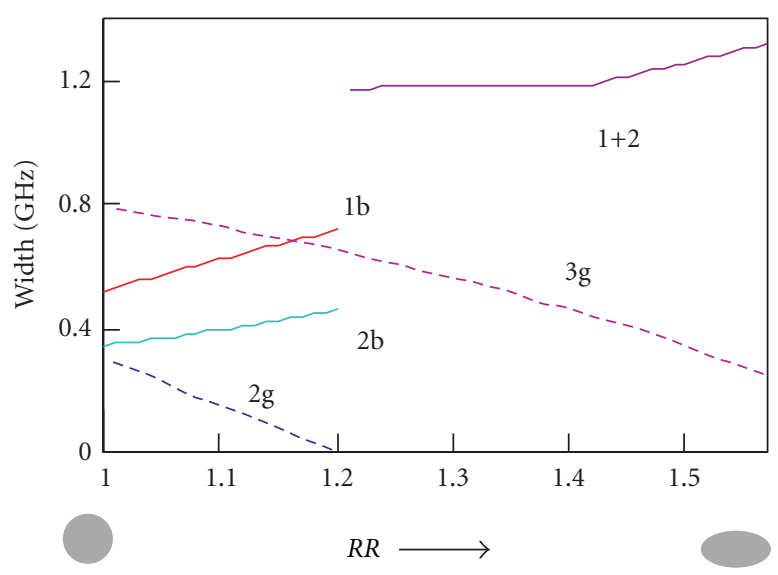

(b)

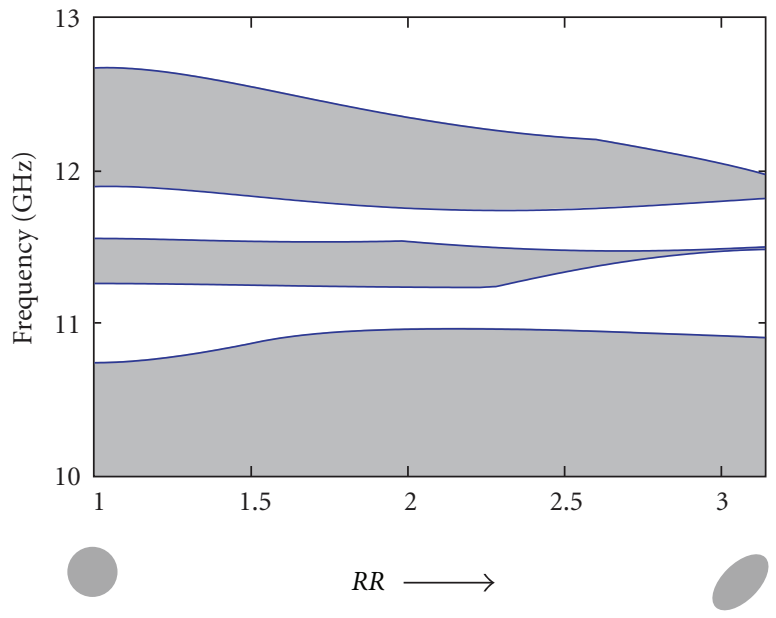

(c)

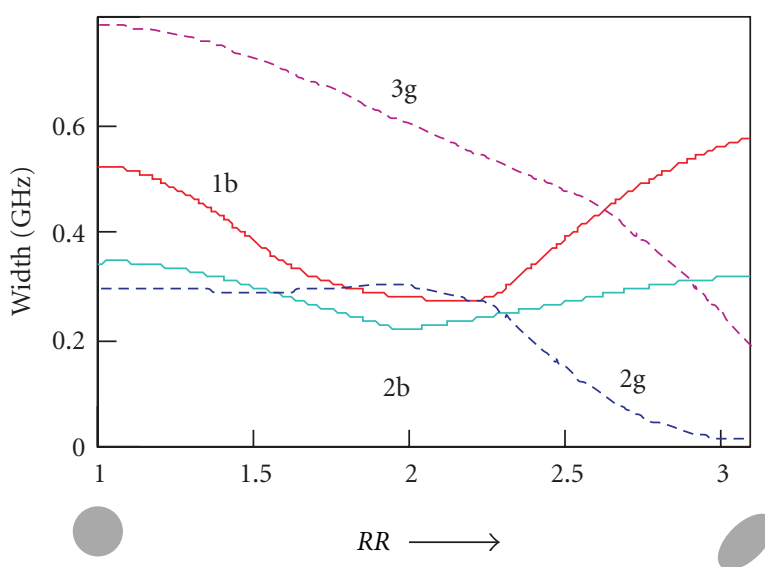

(d)

Figure 4: The same as in Figure 3, but for filling fraction 0.5.

frequency range in which magnonic gaps will occur lowers substantially. This finding is consistent with the results obtained for 2D composites with an EuO matrix [28]. However, an entirely different behavior is observed in $3 \mathrm{D}$ magnonic crystals, in which absolute magnonic gaps are destroyed by the magnetostatic interactions [29].

The use of rods in the shape of elliptic cylinders as scattering centers in 2D magnetic composites implies the introduction of two addition structural parameters: the cross-sectional ellipticity of the rods and the angle of their rotation in the plane perpendicular to the rod axis (the plane of spin-wave propagation). In contrast to the lattice constant, a change of which will strongly modify the spinwave spectrum, these new parameters allow fine tuning of the width and position of the bands and gaps. For specific in-plane rotation angles, changing the rod ellipticity will modify the position of a band without changing its width or cause two adjacent bands to shrink substantially without affecting the width of the gap between them. Thus, an appropriate use of rods of elliptical cross section offers additional possibilities in the design of spin-wave filters with precisely adjusted passband.

\section{Acknowledgments}

Those results were presented at 18th International Conference on Composite Materials, Jeju Island, Korea. The research leading to these results has received funding from the European Community's Seventh Framework Programme (FP7/2007-2013) under Grant Agreement no. 228673 for MAGNONICS project.

\section{References}

[1] A. V. Chumak, A. A. Serga, B. Hillebrands, and M. P. Kostylev, "Scattering of backward spin waves in a one-dimensional magnonic crystal," Applied Physics Letters, vol. 93, no. 2, Article ID 022508, 2008.

[2] Z. K. Wang, V. L. Zhang, H. S. Lim et al., "Observation of frequency band gaps in a one-dimensional nanostructured 
magnonic crystal," Applied Physics Letters, vol. 94, no. 8, Article ID 083112, 2009.

[3] S. L. Vysotskii, S. A. Nikitov, E. S. Pavlov, and Y. A. Filimonov, "The spectrum of the spin-wave excitations of the tangentially magnetized 2D hexagonal ferrite magnonic crystal," Journal of Communications Technology and Electronics, vol. 55, no. 7, pp. 800-809, 2010.

[4] S. Neusser, G. Duerr, H. G. Bauer et al., "Anisotropic propagation and damping of spin waves in a nanopatterned antidot lattice," Physical Review Letters, vol. 105, no. 6, Article ID 067208, 2010.

[5] Y. Cao, G. Yun, X. Liang, and N. Bai, "Band structures of two-dimensional magnonic crystals with different shapes and arrangements of scatterers," Journal of Physics D, vol. 43, no. 30, Article ID 305005, 2010.

[6] J. W. Kłos, M. Krawczyk, and M. Sokolovskyy, "Bulk and edge modes in two-dimensional magnonic crystal slab," Journal of Applied Physics, vol. 109, no. 7, Article ID 07D311, 2011.

[7] R. Zivieri, S. Tacchi, F. Montoncello et al., "Bragg diffraction of spin waves from a two-dimensional antidot lattice," Physical Review B, vol. 85, no. 1, Article ID 012403, pp. 1-6, 2012.

[8] S. A. Nikitov, P. Tailhades, and C. S. Tsai, "Spin waves in periodic magnetic structures-magnonic crystals," Journal of Magnetism and Magnetic Materials, vol. 236, no. 3, pp. 320330, 2001.

[9] M. Krawczyk and H. Puszkarski, "Magnonic excitations versus three-dimensional structural periodicity in magnetic composites," Crystal Research and Technology, vol. 41, no. 6, pp. 547552, 2006.

[10] S. Neusser, H. G. Bauer, G. Duerr et al., "Tunable metamaterial response of a Ni80Fe20 antidot lattice for spin waves," Physical Review B, vol. 84, no. 18, Article ID 184411, 2011.

[11] S. Tacchi, F. Montoncello, M. Madami et al., "Band diagram of spinwaves in a two-dimensional magnonic crystal," Physical Review Letters, vol. 107, no. 12, Article ID 127204, pp. 1-5, 2011.

[12] J. Topp, D. Heitmann, M. P. Kostylev, and D. Grundler, "Making a reconfigurable artificial crystal by ordering bistable magnetic nanowires," Physical Review Letters, vol. 104, no. 20, Article ID 207205, 2010.

[13] S. Tacchi, M. Madami, G. Gubbiotti et al., "Analysis of collective spin-wave modes at different points within the hysteresis loop of a one-dimensional magnonic crystal comprising alternative-width nanostripes," Physical Review B, vol. 82, no. 18, Article ID 184408, 2010.

[14] S. Tacchi, M. Madami, G. Gubbiotti et al., "Anisotropic dynamical coupling for propagating collective modes in a twodimensional magnonic crystal consisting of interacting squared nanodots," Physical Review B, vol. 82, no. 2, Article ID 024401, 2010.

[15] G. Duerr, M. Madami, S. Neusser et al., "Spatial control of spin-wave modes in Ni80Fe20 antidot lattices by embedded Co nanodisks," Applied Physics Letters, vol. 99, no. 20, Article ID 202502, 2011.

[16] S. Neusser, G. Duerr, S. Tacchi et al., "Magnonic minibands in antidot lattices with large spin-wave propagation velocities," Physical Review B, vol. 84, no. 9, Article ID 094454, pp. 1-9, 2011.

[17] V. V. Kruglyak, S. O. Demokritov, and D. Grundler, "Magnonics," Journal of Physics D, vol. 43, no. 26, Article ID 264001, 2010.

[18] S. Neusser and D. Grundler, "Magnonics: spin waves on the nanoscale," Advanced Materials, vol. 21, no. 28, pp. 2927-2932, 2009.
[19] S. Choi, K. S. Lee, K. Y. Guslienko, and S. K. Kim, "Strong radiation of spin waves by core reversal of a magnetic vortex and their wave behaviors in magnetic nanowire waveguides," Physical Review Letters, vol. 98, no. 8, Article ID 087205, 2007.

[20] A. B. Ustinov, A. V. Drozdovskii, and B. A. Kalinikos, "Multifunctional nonlinear magnonic devices for microwave signal processing," Applied Physics Letters, vol. 96, no. 14, Article ID 142513, 2010.

[21] F. S. Ma, H. S. Lim, Z. K. Wang, S. N. Piramanayagam, S. C. $\mathrm{Ng}$, and M. H. Kuok, "Micromagnetic study of spin wave propagation in bicomponent magnonic crystal waveguides," Applied Physics Letters, vol. 98, no. 15, Article ID 153107, 2011.

[22] H. T. Nembach, J. M. Shaw, T. J. Silva et al., "Effects of shape distortions and imperfections on mode frequencies and collective linewidths in nanomagnets," Physical Review B, vol. 83, no. 9, Article ID 094427, 2011.

[23] S. Mamica and M. Krawczyk, "Tuning of the spin-wave band structure in $2 \mathrm{~d}$ magnetic composites," in Proceedings of the 18th International Conference on Composite Materials, P4-34, Jeju Island, Rpublic of Korea, 2011.

[24] M. Krawczyk, J. Klos, M. L. Sokolovskyy, and S. Mamica, "Materials optimization of the magnonic gap in threedimensional magnonic crystals with spheres in hexagonal structure," Journal of Applied Physics, vol. 108, no. 9, Article ID 093909, 2010.

[25] C. A. F. Vaz, J. A. C. Bland, and G. Lauhoff, "Magnetism in ultrathin film structures," Reports on Progress in Physics, vol. 71, no. 5, Article ID 056501, 2008.

[26] V. S. Tkachenko, V. V. Kruglyak, and A. N. Kuchko, "Spectrum and reflection of spin waves in magnonic crystals with different interface profiles," Physical Review B, vol. 81, no. 2, Article ID 024425, 2010.

[27] M. Krawczyk, "Magnetostatic waves in one-dimensional magnonic crystals with magnetic and nonmagnetic components," IEEE Transactions on Magnetics, vol. 44, no. 11, pp. 2854-2857, 2008.

[28] J. O. Vasseur, L. Dobrzynski, B. Djafari-Rouhani, and H. Puszkarski, "Magnon band structure of periodic composites," Physical Review B, vol. 54, no. 2, pp. 1043-1049, 1996.

[29] M. Krawczyk and H. Puszkarski, "Plane-wave theory of threedimensional magnonic crystals," Physical Review B, vol. 77, no. 5, Article ID 054437, 2008. 

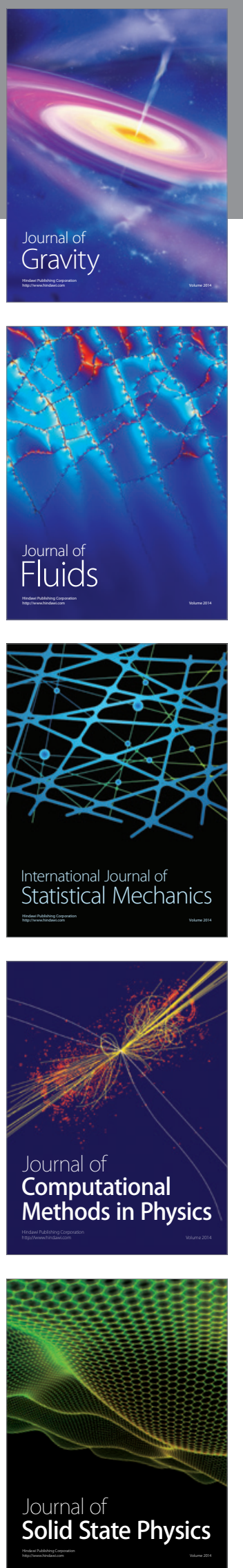

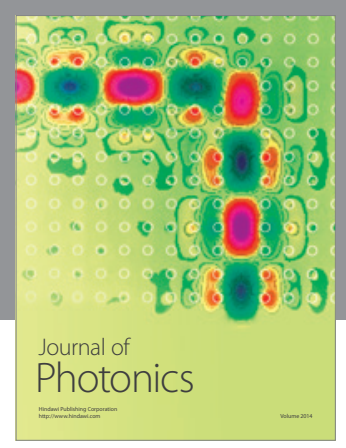

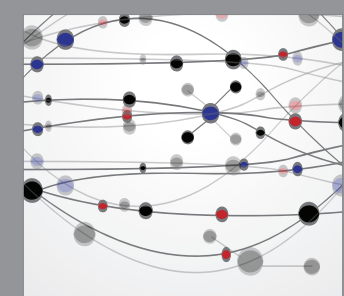

The Scientific World Journal
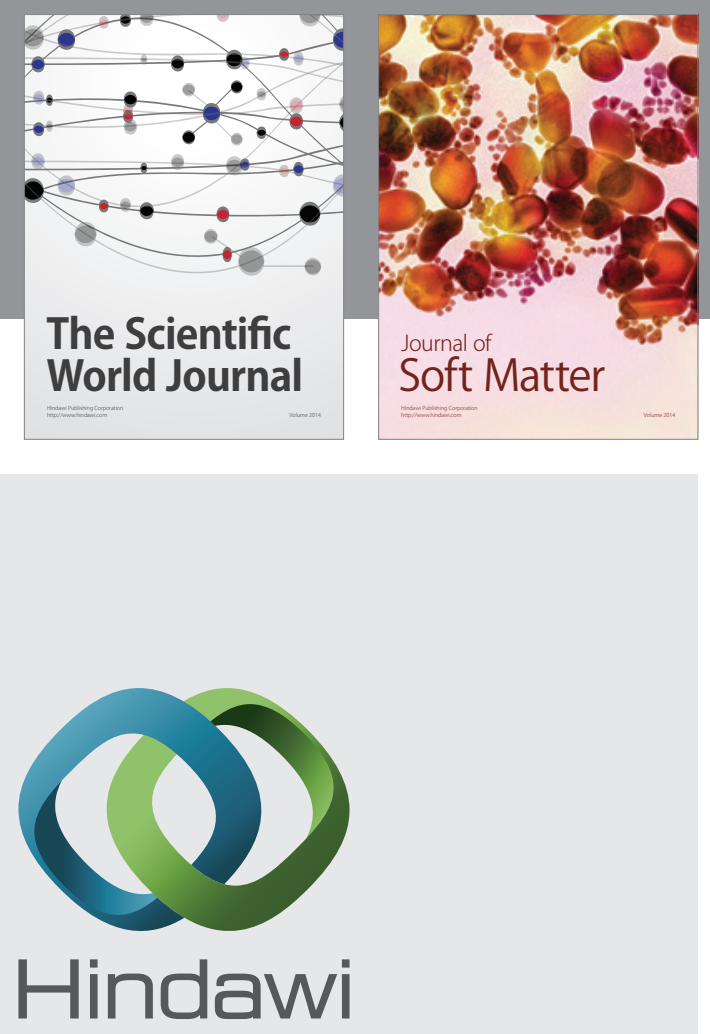

Submit your manuscripts at

http://www.hindawi.com
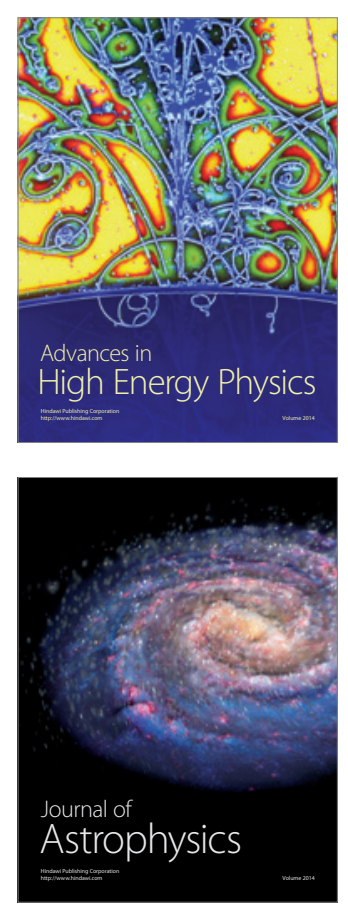
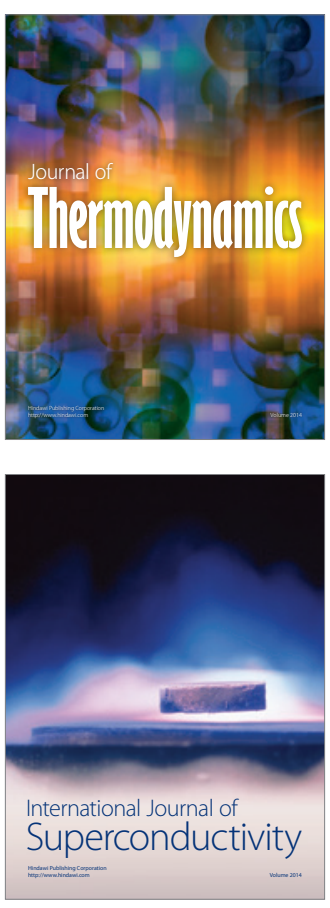
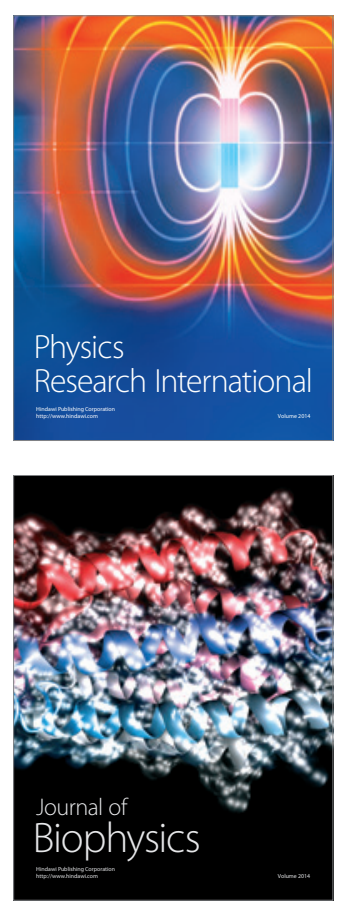
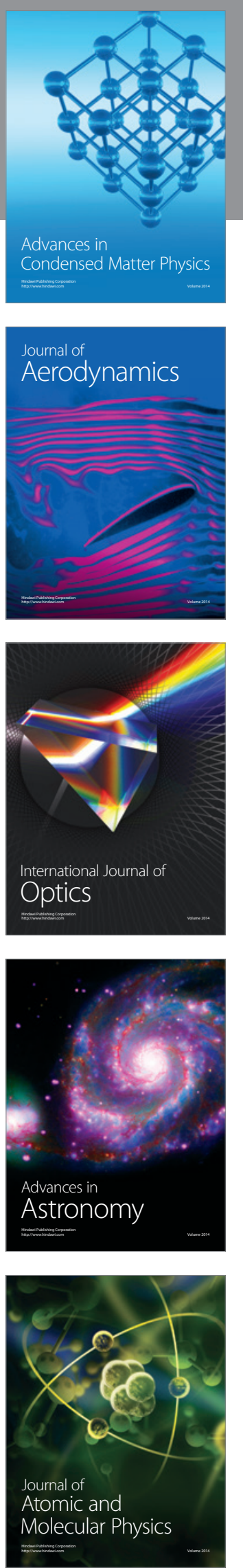